\title{
Radiation Tolerance of LuAG:Ce and YAG:Ce Crystals under High Levels of Gamma- and Proton-Irradiation
}

\begin{tabular}{|r|l|}
\hline Journal: & IEEE Transactions on Nuclear Science \\
\hline Manuscript ID: & TNS-00475-2015 \\
\hline Danuscript Type: & SCINT 2015 \\
\hline Complete List of Authors: & $\begin{array}{l}\text { Lucchini, Marco; CERN, PH-CMX } \\
\text { Pauwels, Kristof; CERN, PH-CMX } \\
\text { Auffray, Etiennette; CERN, PH-CMX } \\
\text { Ochesanu, Silvia; Crytur, } \\
\text { Blazek, Karel; Crytur, }\end{array}$ \\
\hline Standard Key Words: & Radiation hardness, Calorimeters, Crystal characterization \\
\hline & \\
\hline
\end{tabular}




\title{
Radiation Tolerance of LuAG:Ce and YAG:Ce Crystals under High Levels of Gamma- and Proton-Irradiation
}

\author{
M.T. Lucchini, K. Pauwels, K. Blazek, S. Ochesanu and E. Auffray, Member, IEEE
}

\begin{abstract}
The extremely harsh conditions, in which the detectors will have to operate during the High Luminosity phase of the Large Hadron Collider at CERN, set stringent requirements on the properties of the scintillators which can be used. Among different scintillating materials under study, inorganic crystals such as LuAG:Ce and YAG:Ce represent good candidates for such application. A detailed investigation of the radiation hardness of LuAG:Ce and YAG:Ce crystal samples $\left(1 \times 1 \times 1 \mathbf{c m}^{3}\right.$ cubes) produced by Crytur is presented in this study. Given their potential in many calorimeter designs, YAG:Ce samples with high aspect ratio $\left(1 \times 1 \times 14 \mathbf{c m}^{3}\right)$ have also been tested. Optical and scintillating properties of the samples were studied before and after irradiation with different sources and at different intensities. Irradiation with gamma-rays to the doses of 1 and $100 \mathrm{kGy}$ and with $24 \mathrm{GeV}$ protons up to an integrated fluence of $10^{14} \mathrm{~cm}^{-2}$ were performed at CERN. The scintillating properties of the crystals, as emission and excitation spectra and light yield remained unchanged after irradiation and only small levels of induced absorption were observed. The results obtained in this test confirm the potential of LuAG:Ce and YAG:Ce crystals as good candidates for calorimetry applications in future high energy physics experiments.
\end{abstract}

Index Terms - Radiation hardness, inorganic scintillators, LuAG, YAG, proton fluences, gamma-irradiation.

\section{Motivations}

$\mathbf{T}$ HE extremely high levels of both ionizing and hadronic radiation in which detectors will have to operate during the High Luminosity phase of the Large Hadron Collider (HLLHC) at CERN, will require an excellent radiation hardness of all its components. Such operating conditions set stringent requirements on the properties of the scintillators which can be used for calorimetry applications.

Beside high density, fast response and high light yield, the scintillators have to withstand very high levels of radiation, especially in the forward region of the detectors. For instance, in the CMS forward calorimeters, ionizing doses and hadron fluences will reach up to $300 \mathrm{kGy}$ (at a dose rate of 30 $\mathrm{Gy} / \mathrm{h})$ and $2 \times 10^{14} \mathrm{~cm}^{-2}$, respectively, at $|\eta|=2.6$ [1]. Given their high light yield and high density, Aluminum-garnet crystals such as $\mathrm{Lu}_{3} \mathrm{Al}_{5} \mathrm{O}_{12}$ : $\mathrm{Ce}$ (LuAG:Ce) and $\mathrm{Y}_{3} \mathrm{Al}_{5} \mathrm{O}_{12}$ : $\mathrm{Ce}$ (YAG:Ce) represent good candidates for calorimetry applications and it is important to evaluate precisely their radiation tolerance.

Manuscript received June 10, 2015.

M. Lucchini, K. Pauwels and E. Auffray are with CERN, Geneva, Switzerland.

K. Blazek and S. Ochesanu are with Crytur, Turnov, Czech Republic.
Radiation hardness of inorganic crystals strongly depends on the purity of the raw material as on the growth parameters. The presence of impurities can lead to the creation of colorcenters which degrade the light transmission after gammairradiation [2]. In addition, hadronic radiation can produce displacements of ions in the crystal lattice leading to additional loss of transparency [3]. It is therefore of crucial importance, to evaluate the radiation tolerance under both type of radiation. To this extent we present in this study the results of gamma and proton-irradiations on LuAG:Ce and YAG:Ce crystals.

\section{Crystals Characterization}

The LuAG:Ce and YAG:Ce samples were grown by Crytur using Czochralski technique and then cut and polished. Two geometries with extremely different aspect ratios have been studied in this work: cubic samples of $10 \times 10 \times 10 \mathrm{~mm}^{3}$ and fiber-like samples of $1 \times 1 \times 140 \mathrm{~mm}^{3}$ as shown in Fig. 1 and Fig. 2 respectively. The four YAG:Ce and LuAG:Ce cubic crystal samples (LuAG c1, LuAG c2, YAG c1, YAG c2) were fully characterized at CERN by measuring their scintillation properties before and after irradiation. The light yield was measured using a $\mathrm{Cs}^{137} \gamma$-source and coupling the polished face of the sample to a Hamamastsu R2059 PMT [4] with Q.E. of 9\% and 6.5\% for the LuAG:Ce and YAG:Ce emission spectra respectively. Results of light yield measurements obtained with grease coupling and without wrapping are reported in Table I. Excitation/emission spectra and transmission curves were measured over the range of wavelength of 300-700 nm using a dedicated spectrophotometer. A measurement of the scintillation time profiles was also performed, by averaging about 10000 scintillation pulses, to extract an estimation of the decay time components. A summary of these measurements is reported in Table I and shows consistent agreement with previous results [5], [6].

TABLE I

CRYSTAL SAMPLES SCINTILLATION PROPERTIES.

\begin{tabular}{|c|c|c|c|c|}
\hline & $\begin{array}{c}\text { Emission Peak } \\
{[\mathbf{n m}]}\end{array}$ & $\begin{array}{c}\text { Light Yield } \\
{[\mathbf{p h} / \mathbf{M e V}]}\end{array}$ & $\begin{array}{c}\tau_{1}\left(A_{1}\right) \\
{[\mathrm{ns}]}\end{array}$ & $\begin{array}{c}\tau_{2}\left(A_{2}\right) \\
{[\mathrm{ns}]}\end{array}$ \\
\hline LuAG:Ce c1 & 520 & 13500 & $58(0.78)$ & $850(0.22)$ \\
YAG:Ce c1 & 540 & 17800 & $97(0.92)$ & $330(0.08)$ \\
\hline
\end{tabular}

\section{EXPERIMENTAL PROCEDURE}

Optical and scintillation properties of the samples have been studied before and after irradiation to evaluate damage effects 
Fig. 1. LuAG:Ce (left) and YAG:Ce (right) cubic samples of $10 \times 10 \times$ $10 \mathrm{~mm}^{3}$, polished on two parallel faces.

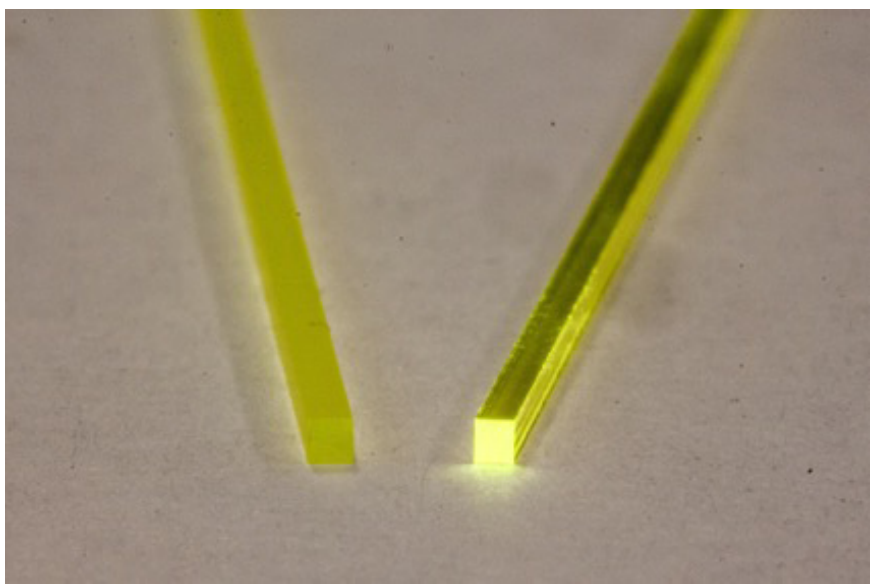

Fig. 2. YAG:Ce high aspect ratio samples of $1 \times 1 \times 140 \mathrm{~mm}^{3}$, before (left) and after the polishing process (right). Left sample shows the large diffusion occuring at the lateral surface in case of no polishing.

on the crystal performance under several aspects.

As a first step, irradiation with $\gamma$-rays from a Co-60 source was perfomed at Dagneux-irradiation facility [7]. LuAG and YAG samples have been irradiatied at a dose rate of $\sim 1 \mathrm{kGy} / \mathrm{h}$ up to $1 \mathrm{kGy}$ of integrated dose. After measuring their transmission, the same samples have been further irradiated to the total dose of $100 \mathrm{kGy}$ which is closer to radiation levels expected in the forward region of detectors during HL-LHC phase. In both cases, the transmission measurement was performed about 2 hours after irradiation, limiting as much as possible the effect of eventual recovery at room temperature.

In a second moment, LuAG and YAG samples have been irradiated with $24 \mathrm{GeV}$ protons provided by the IRRAD facility at CERN Proton Synchrotron [8]. The size of the proton beam is about $1 \mathrm{~cm}$ wide which allows to uniformly irradiate the whole volume of the crystals. A total integrated fluence of $10^{14} \mathrm{p} / \mathrm{cm}^{-2}$ was achieved at a proton flux of $1.8 \times 10^{13} \mathrm{p} / \mathrm{cm}^{2} / \mathrm{h}$. After a period of about $1 \mathrm{month}$, required for the radioactive cool down of the elements, the samples were re-measured in the laboratory.

\section{Results on Cubic Samples}

Transmission curves were measured before $\left(T_{b}\right)$ and after each irradiation $\left(T_{a}\right)$ in the $300-700 \mathrm{~nm}$ range. The induced absorption coefficient has been calculated according to:

$$
\mu_{i n d}=\frac{1}{L} \ln \left(\frac{T_{b}}{T_{a}}\right)
$$

where $L$ is the crystal length along the light beam direction, which for the samples of this study corresponds to $1 \mathrm{~cm}$. The uncertainty on the estimated $\mu_{i n d}$ is affected by fluctuations in the spectrophotemeter response and by the precision in the alignment of the crystal faces with respect to the light beam. These have been estimated to produce an error of about $\pm 0.3 \mathrm{~m}^{-1}$ on the induced absorption coefficient.

The results obtained after gamma irradiation on samples LuAG:Ce c1 and YAG:Ce c1 are reported in Fig. 3 and Fig. 4 respectively. A loss of light transmission is observed in the low wavelength region $(310-370 \mathrm{~nm})$ whereas at the emission peak (520-540 nm), induced absorption is close to zero. Similar results were obtained after the proton-irradiation on samples LuAG:Ce c2 and YAG:Ce c2 as reported in Fig. 5 and Fig. 6 respectively. No modification of scintillation properties, such as light yield, emission spectrum or pulse shape, was observed after gamma-rays and protons irradiations. This is consistent with the negligible loss of light in the region of scintillation emission.

The value of $\mu_{\text {ind }}$ calculated for different samples and different irradiations are reported in Table II.

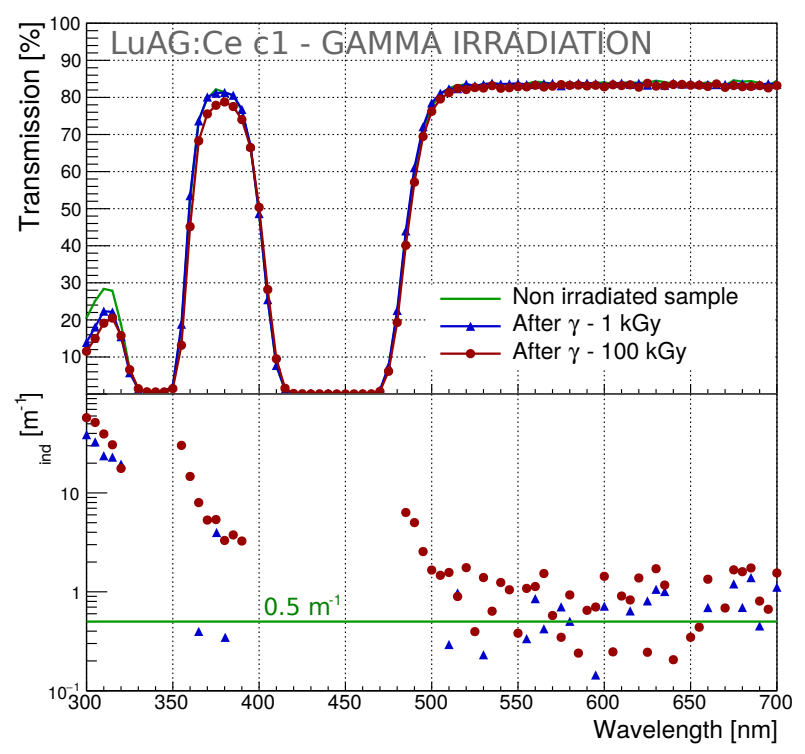

Fig. 3. Top: Transmission curves for LuAG:Ce c1 sample before irradiation (green line), after $1 \mathrm{kGy}$ (blue triangles) and $100 \mathrm{kGy}$ (red circles) of $\gamma$ irradiation. Bottom: The calculated induced absorption coefficient $\mu_{\text {ind }}$ is reported for both irradiations, as a function of wavelength. Green line at $0.5 \mathrm{~m}^{-1}$ is drawn as reference value.

\section{Results on High Aspect Ratio Samples}

Because of the small cross section $\left(1 \times 1 \mathrm{~mm}^{2}\right)$ of the samples and their extremely high aspect ratio, a measurement 
Fig. 4. Top: Transmission curves for YAG:Ce c1 crystals before irradiation (green line), after $1 \mathrm{kGy}$ (blue triangles) and $100 \mathrm{kGy}$ (red circles) of $\gamma$ irradiation. Bottom: The calculated induced absorption coefficient $\mu_{i n d}$ is reported for both irradiations, as a function of wavelength. Green line at $0.5 \mathrm{~m}^{-1}$ is drawn as reference value.

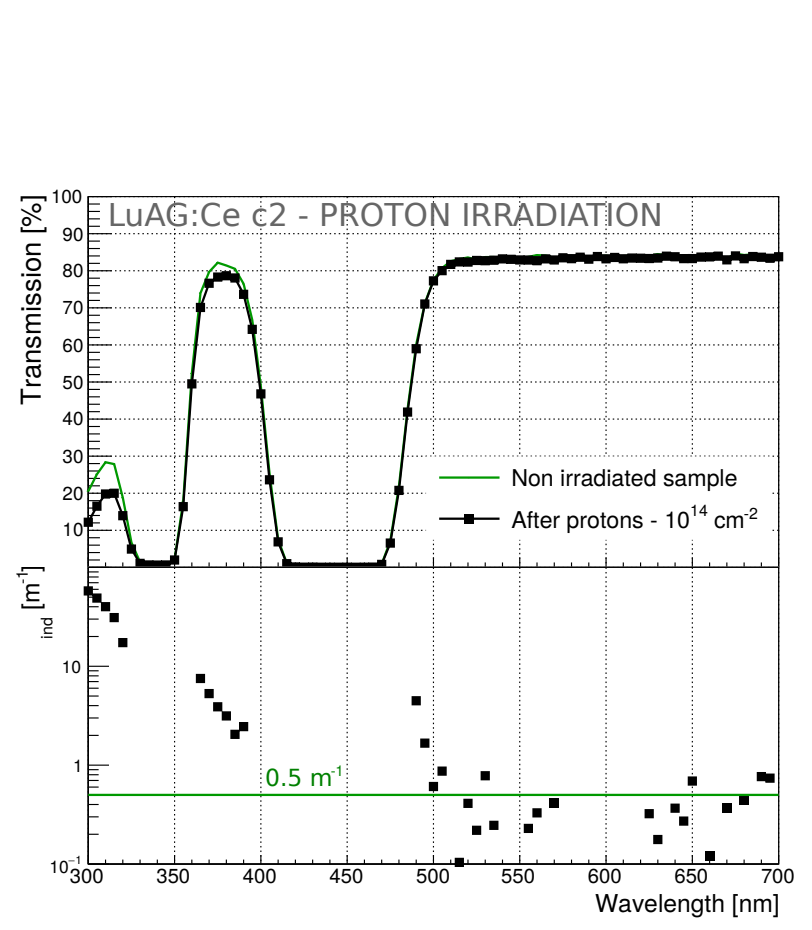

Fig. 5. Top: Transmission curves for LuAG:Ce $\mathrm{c} 2$ crystals before irradiation (green line) and after irradiation with protons (black squared dots). Bottom: The calculated induced absorption coefficient $\mu_{\text {ind }}$ is reported for both irradiations, as a function of wavelength. Green line at $0.5 \mathrm{~m}^{-1}$ is drawn

as reference value.

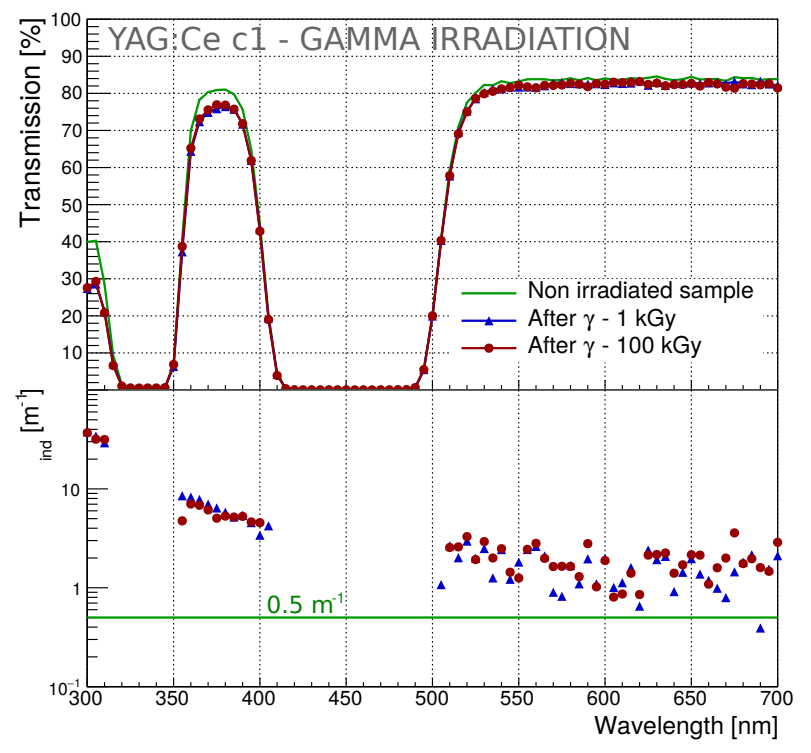

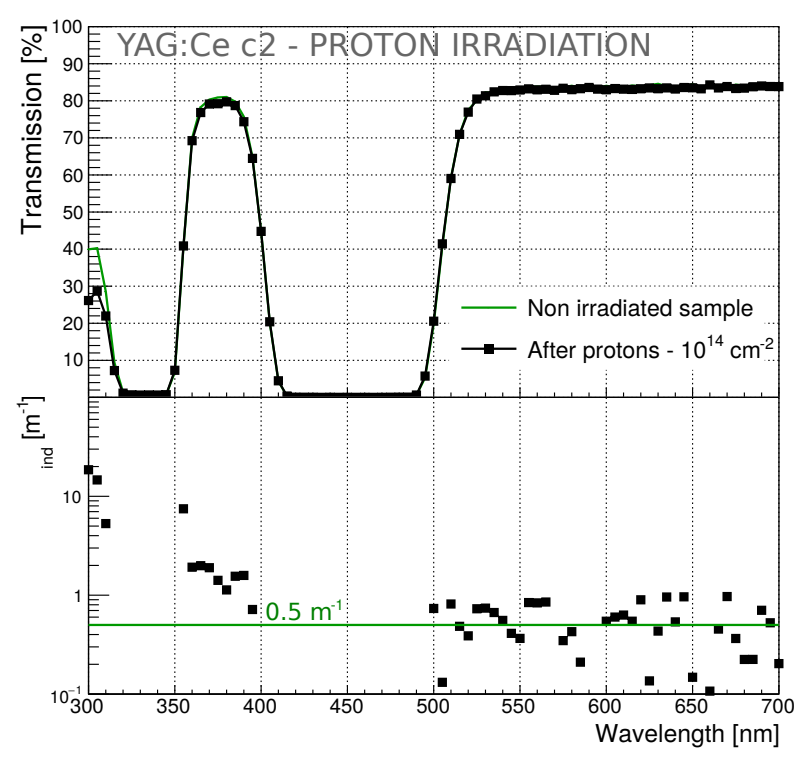

Fig. 6. Top: Transmission curves for YAG:Ce $\mathrm{c} 2$ crystals before irradiation (green line) and after irradiation with protons (black squared dots). Bottom: The calculated induced absorption coefficient $\mu_{\text {ind }}$ is reported for both irradiations, as a function of wavelength. Green line at $0.5 \mathrm{~m}^{-1}$ is drawn as reference value.

TABLE II

INDUCED ABSORPTION MEASURED ON CUBIC SAMPLES

\begin{tabular}{|c|c|c|c|}
\hline $\begin{array}{c}\text { Crystal } \\
\text { ID }\end{array}$ & $\begin{array}{c}\mu_{\text {ind }} \\
\text { after 1 kGy }\end{array}$ & $\begin{array}{c}\mu_{\text {ind }} \\
\text { after 100 kGy }\end{array}$ & $\begin{array}{c}\mu_{\text {ind }} \\
\text { after protons }\end{array}$ \\
\hline \hline YAG:Ce c1 & $1.1 \pm 0.3 \mathrm{~m}^{-1}$ & $2.1 \pm 0.3 \mathrm{~m}^{-1}$ & - \\
LuAG:Ce c1 & $0.5 \pm 0.3 \mathrm{~m}^{-1}$ & $1.6 \pm 0.3 \mathrm{~m}^{-1}$ & - \\
\hline YAG:Ce c2 & $0.6 \pm 0.3 \mathrm{~m}^{-1}$ & - & $0.6 \pm 0.3 \mathrm{~m}^{-1}$ \\
LuAG:Ce c2 & $0.4 \pm 0.3 \mathrm{~m}^{-1}$ & - & $0.5 \pm 0.3 \mathrm{~m}^{-1}$ \\
\hline
\end{tabular}

of direct light transmission is very difficult to perform with standard spectrophotometers. At the same time, in crystals with such geometry the importance of lateral surface state becomes of crucial importance for an optimal propagation of the scintillation light along the sample.

For this reason a measurement of attenuation length was chosen as the main parameter for the characterization of these fiber-like samples. Such measurement is performed by illuminating the crystal with a LED at different positions along its longitudinal axis, as shown in Fig. 7, and monitoring the light output at both crystal ends using SiPMs. The double-side readout of the light allows to cross check the measurement and to correct in case of small variations of the LED intensity, as discussed in [9].

A small deterioration of attenuation curves due to $\gamma$ irradiation was observed as shown in Fig. 8. However, a comparison of the damage effect after integrated doses of 1 and $100 \mathrm{kGy}$ shows a non linear behavior of the induced attenuation length, suggesting that damage effects are saturating at high doses.

The result of proton-irradiation on a YAG:Ce high aspect ratio crystal is reported in Fig. 9. A very small degradation 


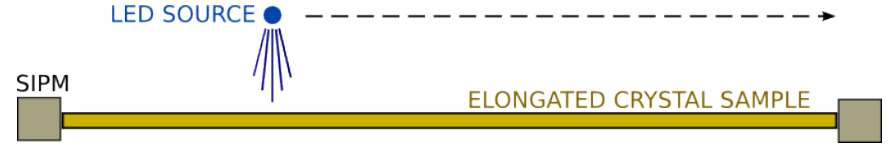

Fig. 7. Schematic of the setup used to measure attenuation curves of elongated samples. A SiPM with $3 \times 3 \mathrm{~mm}^{3}$ active area is located at each end of the sample. The pulsed LED light source on top of the sample, at about $1 \mathrm{~cm}$ distance, is moved along the longitudinal axis of the crystal.

of the attenuation length, comparable with the effect of low $\gamma$-rays dose, is observed.

To quantify the radiation damage effects, the attenuation curves have been fitted with the exponential curve,

$$
I=I_{0} \cdot e^{-d / L_{a t t}}
$$

where the normalization constant $I_{0}$ corresponds to the light signal when the LED illuminates the crystal close to the SiPM, at a distance $d=0$, and the parameter $L_{a t t}$ represents the attenuation length of the sample. In Table III we report the values of $L_{\text {att }}$ of the samples tested before and after different types of irradiations. The YAG:Ce sample f3 was shorter (100 mm only) and thus only attenuation curve within a restricted range was measured. Due to the shorter length the light collection inside such crystal behaves in a different way, being less sensitive to surface defects and intrinsic absorption, and can explain the difference in the initial attenuation length reported in Table III $(122 \mathrm{~cm}$ instead of $60 \mathrm{~cm}$ as measured on longer samples).

TABLE III

attenuation Lengths of High Aspect Ratio Samples

\begin{tabular}{|c|c|c|c|c|}
\hline $\begin{array}{c}\text { Crystal } \\
\text { (ID) }\end{array}$ & $\begin{array}{c}L_{a t t} \\
\text { before }\end{array}$ & $\begin{array}{c}L_{a t t} \\
\text { after 1 kGy }\end{array}$ & $\begin{array}{c}L_{a t t} \\
\text { after } 100 \mathrm{kGy}\end{array}$ & $\begin{array}{c}L_{a t t} \\
\text { after protons }\end{array}$ \\
\hline \hline YAG f1 & $60 \pm 1 \mathrm{~cm}$ & $40 \pm 1 \mathrm{~cm}$ & $29 \pm 1 \mathrm{~cm}$ & - \\
YAG f2 & $61 \pm 1 \mathrm{~cm}$ & $43 \pm 1 \mathrm{~cm}$ & $28 \pm 1 \mathrm{~cm}$ & - \\
YAG f3 & $122 \pm 2 \mathrm{~cm}$ & - & - & $64 \pm 1 \mathrm{~cm}$ \\
\hline
\end{tabular}

\section{DISCUSSION}

The comparison of scintillation and optical properties of LuAG:Ce and YAG:Ce samples before and after irradiation up to high doses and fluences demonstrates a very good radiation tolerance of these garnet crystals.

The evolution of transmission curves of cubic samples and the change in attenuation length of high aspect ratio crystals represent complementary and independent measurements of the radiation damage effects. In both cases the results yield a consistent quantification of the light transmission loss which can be interpreted in terms of an induced absorption coefficient ranging from 0.4 to $2 \mathrm{~m}^{-1}$ depending on the crystal and irradiation type as reported in Table II.

A first important result of this irradiation test, is the proof that no specific absorption peak, i.e. creation of color centers, is observed in the wavelength range of emission from 500 to $700 \mathrm{~nm}$. This demonstrates the very good quality of the raw material and the potential radiation hardness of these crystals.

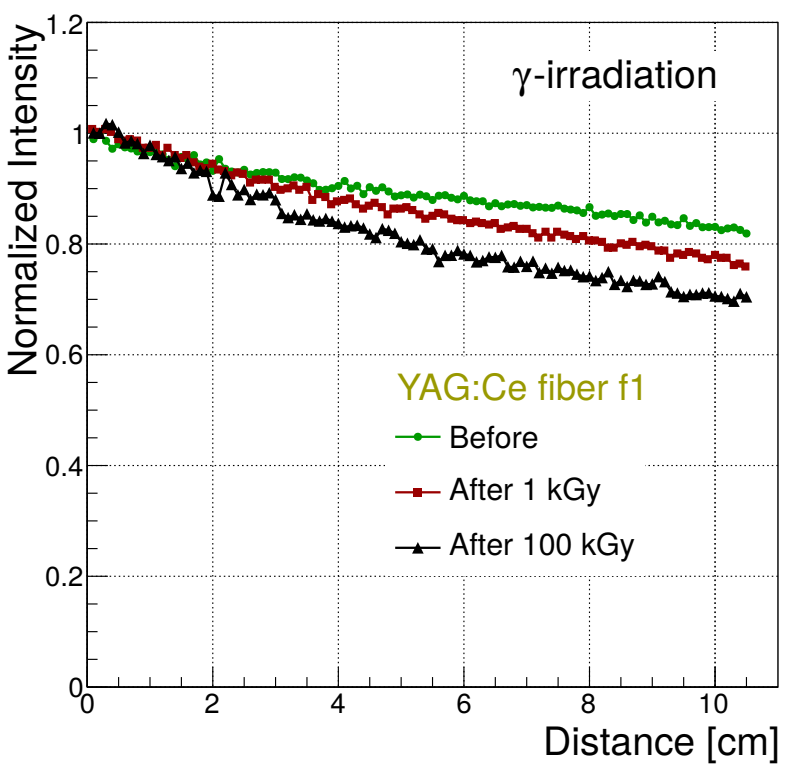

Fig. 8. Attenuation curves of YAG:Ce elongated samples before (green circles) and after gamma-irradiations to $1 \mathrm{kGy}$ (red squares) and $100 \mathrm{kGy}$ (black triangles).

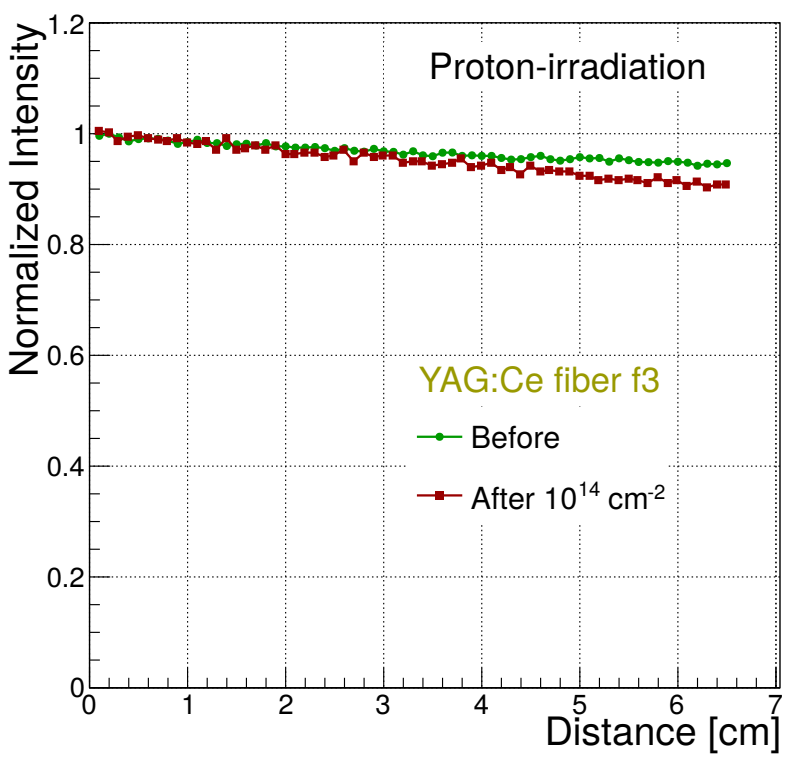

Fig. 9. Attenuation curves of YAG:Ce elongated samples before (green circles) and after proton-irradiation (red squares). 
The irradiation with $\gamma$-rays at different doses also gives indications that transmission loss in not linearly proportional to the the integrated dose and that saturation effects occurs beyond $1 \mathrm{kGy}$.

\section{CONCLUSIONS AND OUTLOOK}

The results obtained with this irradiation campaign demonstrate a good radiation hardness for LuAG:Ce and YAG:Ce crystals, making them suitable for calorimetry applications in detectors for future high energy physics experiments. In particular, high aspect ratio crystals, as those characterized in this study, represent a powerful tool for calorimeter designs due to their extremely flexible geometry as discussed in [10].

The loss of transmission observed at low wavelengths, however, indicates that some creation of defects occurs as consequence of both $\gamma$ and proton irradiation. Although, at such wavelengths this induced absorption does not affect the scintillation light, some other effects such as induced phosphorescence might occur as reported in [13]. Additional studies, such as thermo-stimulated luminescence (TSL), should be performed to further investigates the nature of such defects.

Further R\&D studies are also ongoing to optimize this technology for calorimetry applications. The addition of codopants, such as divalent cations like $\mathrm{Ca}^{2+}$ or $\mathrm{Mg}^{2+}$, is a possible way to improve the radiation hardness of garnet based crystal as discussed in [11]. At the same time, codopants can be used to reduce the long decay time components of the scintillation as demonstrated for the case $\mathrm{Mg}$ - ions for codoping LuAG:Ce crystals [12]. These two, fast decay time and improved radiation tolerance are key parameters for any scintillators to be used in high radiation environment such as detectors in HL-LHC.

\section{ACKNOWLEDGMENT}

This work was carried on in the frame of the Crystal Clear and CMS collaborations and as a part of the project RANUSTD TE01020445, supported by Technology Agency of Czech Republic. This study is also supported by the European Union's Horizon 2020 research and innovation programme under the Marie-Curie grant agreement No. 644260.

\section{REFERENCES}

[1] M. Lucchini et al, Evolution of the response of the CMS ECAL and possible design options for calorimetry at the High Luminosity LHC, Nuclear Science Symposium and Medical Imaging Conference (NSS/MIC), 2013 IEEE , (2013).

[2] M.V. Derdzyan et al, Radiation hardness of LuAG:Ce and LuAG:Pr scintillator crystals, Journal of Crystal Growth, Vol. 361, pp. 212-216, (2012).

[3] P. Adzic et al, Radiation hardness qualification of $\mathrm{PbWO}_{4}$ scintillation crystals for the CMS Electromagnetic Calorimeter, JINST, Vol. 5, pp. 03010, (2009).

[4] Hamamatsu, Hamamatsu R2059 PMT, http://www.hamamatsu.com/us/en/ R2059.html.

[5] C. Dujardin et al, LuAG:Ce fibers for high energy calorimetry, Journal for Applied Physics, Vol. 108, pp. 13510, (2010).

[6] W. Chewpraditkul et al, Scintillation Properties of LuAG:Ce, YAG:Ce and LYSO:Ce Crystals for Gamma-Ray Detection, IEEE Transactions on Nuclear Science, Vol. 56, no. 6, (2009).

[7] D. Pellerin and M. T. Kavanagh, The contract facilities for gamma irradiation at Dagneux, France,
[8] CERN PS Irradiation Facility, http://ps-irrad.web.cern.ch/ps-irrad/ Radiat. Phys. Chem. Vol. 25, pp. 703-708, (1985).

[9] K. Pauwels et al, Single crystalline LuAG fibers for homogeneous dualreadout calorimeters, JINST Vol. 8, pp. 09019, (2013).

[10] M. Lucchini et al, Test beam results with LuAG fibers for next-generation calorimeters, JINST Vol. 8, pp. 10017, (2013).

[11] A. Petrosyan et al, A study of radiation effects on LuAG:Ce(Pr) coactivated with Ca, Journal of Crystal Growth, to be published, (2015).

[12] M. Nikl et al, Defect Engineering in Ce-Doped Aluminum Garnet Single Crystal Scintillators, Crystal Growth and Design, Vol. 9, pp. 4827-4833, (2014).

[13] E. Auffray et al, Radiation damage of LSO crystals under $\gamma$ - and 24 $\mathrm{GeV}$ protons irradiation, Nuclear Instruments and Methods in Physics Research Section A, Vol. 721, pp. 7682 (2013). 